\title{
Europa ohne Binnengrenzen: eine lange Initialphase
}

Die Debatten um ein „Europa ohne Grenzen“ nach dem Zweiten Weltkrieg müssen vor dem Hintergrund einer langen Linie der Herausbildung von Annahmen über die Vorzüge und Nachteile eines Verzichts auf die Kontrolle von Migrationsbewegungen gesehen werden. Einen zentralen Anknüpfungspunkt bietet die Vorstellung des Liberalismus des 19. Jahrhunderts, eine freie Bewegung von Arbeitskräften und der Verzicht auf Pässe, Visa und Grenzkontrollen sei erforderlich, um den Marktkräften Geltung zu verschaffen und einen Wohlstandsgewinn für alle zu ermöglichen. Die Vereinigten Staaten von Amerika verzichteten bereits seit 1802 darauf, Pässe bei einer Einreise zu verlangen und Kontrollen durchzuführen. Großbritannien folgte 1836, vornehmlich in den 1850er und 1860er Jahren kamen zahlreiche weitere Staaten West-, Mittel- und Nordeuropas hinzu (Fahrmeir 2001). Das breit rezipierte Rotteck-Welckersche Staatslexikon kommentierte in seiner dritten Auflage 1864 den Wandel in einem Artikel zum Passwesen mit der Bemerkung: „Neuerlich haben denn auch die Regierungen des Continents eingesehen, daß die Paßgesetze mit der bürgerlichen und wirthschaftlichen Freiheit nicht ferner vereinbar sind" (Paßwesen 1864). Interne und grenzüberschreitende Migration galt in dieser Phase hoher wirtschaftlicher Prosperität als Ausweis von Modernität. Alex Dowty (1987, S. 54) beschreibt den Zustand als ,the closest approximation to an open world in modern times."

Übersehen werden darf allerdings nicht, dass nach einigen Jahrzehnten eines abnehmenden Kontrollbedürfnisses und eines Rückgangs der Kontrollintensität seit den 1880/1890er Jahren das Kontrollinteresse sowie die Leistungsfähigkeit der Kontrollinfrastrukturen im euro-atlantischen Raum erneut anwuchsen. Der Aufstieg nationalistischer und rassistischer Vorstellungen trug dazu ebenso bei wie der wachsende politische Einfluss der Arbeiterbewegungen und ihrer Auffassungen über den „Schutz des nationalen Arbeitsmarkts“ vor der Einwanderung von Arbeitskräften, die Löhne unterbieten und Streiks brechen könnten. 
Zunehmend wurden einheimische und zugewanderte Minderheiten in toto als Bedrohung von innerer Sicherheit, Homogenität der Bevölkerung, ökonomischer Stabilität und Kultur der Nation verstanden. Restriktive Minderheitenpolitik und Zuwanderungsschranken gegenüber ausländischen Staatsangehörigen sowie die Aufrichtung formeller bzw. informeller Barrieren der gesellschaftlichen Teilhabe sollten die Gefahren minimieren, die angeblich von Minderheiten ausgingen (Collomp 2003).

Schließlich wurde während des Ersten Weltkriegs im europäischnordatlantischen Raum für den zwischenstaatlichen Personenverkehr der Passund Visumzwang eingeführt sowie der Grenzübertritt massiv erschwert. Diese Neuerung bildete ein Ergebnis des erheblich an Bedeutung gewinnenden Sicherheits- und Kontrollbedürfnisses der Staaten in der Kriegssituation (Torpey 2000, S. 111-117; Lucassen 1998). Die Pflicht, ein Visum vor einer Einreise zu beantragen und damit eine Kontrolle der Migration zu ermöglichen, bevor sie in Gang gesetzt wurde, entwickelte sich nach dem Ersten Weltkrieg zu einem zentralen Instrument der Steuerung von Migrationsbewegungen (Oltmer 2020). Wenngleich das Bedürfnis nach einer Überwachung und Beeinflussung räumlicher Bewegungen hoch war und hoch blieb, schien zahlreichen Regierungen im Europa der Zwischenkriegszeit doch eine sorgsame Beschränkung der Kontrollen geboten: Vielfach wurde argumentiert, Visa-Regelungen würden nicht nur den Reiseverkehr beeinträchtigen, sondern auch die Waren- und Kapitalzirkulation behindern (Oltmer 2005, S. 427-433).

Der Verzicht auf ein Visum in den Beziehungen zwischen einzelnen (aber keineswegs zwischen allen) Staaten galt als Mittel zur Wirtschaftsförderung und als Symbol gegenseitigen Vertrauens, das in einer Privilegierung des Personenverkehrs im Rahmen zahlreicher bilateraler Visa-Abkommen ihren Ausdruck fand. Allerdings verdeutlichten bereits in den 1920er und 1930er Jahren alle Verhandlungen über Visa-Abkommen in Europa, dass sie sich nicht nur auf Regelungen für Geschäftsleute und touristische Reisen beschränken konnten. Sie mussten zwangsläufig die Frage berühren, ob und inwieweit Visafreiheit auch für jene galt, die als Arbeitsmigrantinnen und -migranten im Ankunftsland eine Beschäftigung aufnehmen oder einen Schutzstatus als politisch Verfolgte geltend machen wollten (Mau et al. 2015).

Regelungen zur Beschränkung des Pass- und Visumzwangs sowie zur Erleichterung von Grenzabfertigungen erfolgten im Westen Europas auch nach dem Zweiten Weltkrieg zunächst mithilfe bilateraler Abkommen. Erneut schlossen in den 1950er Jahren zahlreiche Staaten solche Verträge. Belgien, die Niederlande und Luxemburg allerdings verließen den Weg der bloß bilateralen Verständigung 
und vereinbarten 1960 eine Passunion. Diese, aber auch die bilateralen Abkommen über Visafreiheit und eine erleichterte Grenzabfertigung von Menschen und Waren, bildeten eine Voraussetzung für eine multilaterale Abstimmung über den Verzicht auf Grenzkontrollen an den Binnengrenzen, wie sie im Rahmen der EWG diskutiert und schließlich im Schengen-Raum etabliert wurde.

Dem Abschluss multilateraler Abkommen und der Gründung supranationaler Organisationen, die die Migrations- und Grenzpolitik europäischer Staaten seit den 1950er Jahren beeinflussten, ging aber auch eine informelle Standardisierung von Regelungen in Europa voran und begleitete sie bis in die frühen 1970er Jahre: Mit einem Vertrag zwischen Frankreich und Polen im Jahr 1919 setzte der Aufbau eines weit ausgreifenden Geflechts von bilateralen Abkommen zur Anwerbung von (als temporär beschäftigt vorgestellten) Arbeitskräften zwischen agrarisch geprägten Volkswirtschaften im Süden und Osten Europas und den Industriegesellschaften West-, Mittel- und Nordeuropas ein. Bis Anfang der 1970er Jahre wurden rund 120 Anwerbeabkommen geschlossen. Ein Großteil Europas, nach 1945 aber auch Staaten in Asien (Türkei), Nordafrika (Algerien, Marokko, Tunesien) und Westafrika (Elfenbeinküste, Senegal, Togo), waren einbezogen. Zwar bildete der Osten Europas aufgrund der Teilung des Kontinents durch einen „Eisernen Vorhang" nach dem Zweiten Weltkrieg kein Element des Geflechts von Anwerbeabkommen mehr (sieht man von Jugoslawien ab). Zeitgleich aber wuchs die Intensität der zwischenstaatlichen Kooperation vor allem seit Ende der 1950er Jahre im Westen Europas massiv an. Die Hochkonjunktur der Nachkriegsjahrzehnte ließ die Nachfrage nach Arbeitskräften in den Industrieländern ansteigen, die vor allem durch die Zuwanderung aus dem Süden Europas und der Türkei gedeckt werden sollte: 1946 bis 1959 wurden 15 neue bilaterale Wanderungsabkommen geschlossen. 1960 bis 1974 folgten mit 45 weitaus mehr (Rass 2010). Diese Abkommen initiierten, ermöglichten und kanalisierten die Migration zahlreicher Menschen. Allein in die Bundesrepublik Deutschland sollen vom Ende der 1950er Jahre bis 1973 als direkte oder indirekte Folge der Anwerbeaktivitäten 14 Mio. Arbeitskräfte gekommen sein, von denen etwa elf Millionen, also rund $80 \%$, in diesem Zeitraum wieder in ihre Herkunftsländer zurückkehrten (Münz et al. 1997, S. 35-42).

Zwar handelte es sich durchgängig um Verträge zwischen einzelnen Staaten, dennoch kann von einer „Harmonisierung“ migrationspolitischer Regelungen in Europa gesprochen werden: Die Vertragstexte waren in der Regel identisch, ihre Kernformulierungen hatte die Internationale Arbeitsorganisation (ILO) als Organ des Völkerbundes bereits in der Zwischenkriegszeit entwickelt. Wegen der ausgeprägten Konkurrenz um Arbeitskräfte aus dem Süden Europas sahen sich die Industriestaaten nach dem Zweiten Weltkrieg als Zielländer der Arbeitsmigration 
gezwungen, gleichartige Mindeststandards für Beschäftigung, Entlohnung und Unterbringung der Arbeitskräfte zu gewährleisten. Für den in den 1950er Jahren beginnenden Prozess der Herausbildung supranationaler Organisationen in Europa erwiesen sich das Geflecht von Anwerbeverträgen und die Praxis der Anwerbung insofern als unmittelbar von Bedeutung, als unter den Gründungsstaaten der EWG mit Belgien, der Bundesrepublik Deutschland, Frankreich, Luxemburg und den Niederlanden nicht nur die wichtigsten Anwerbeländer vertreten waren, sondern mit Italien auch das bis in die 1960er Jahre wichtigste Herkunftsland von Arbeitsmigrantinnen und Arbeitsmigranten, die sich im System der bilateralen Anwerbeabkommen in Europa bewegten (Oltmer 2014; Oltmer et al. 2012).

Bereits bei den Verhandlungen über eine „Europäische Gemeinschaft für Kohle und Stahl“" (EGKS), die 1951 Belgien, die Bundesrepublik Deutschland, Frankreich, Italien, Luxemburg und die Niederlande zur gemeinsamen Förderung und Kontrolle der Kohle- und Stahlerzeugung sowie der zollfreien Vermarktung der Produkte untereinander gründeten, sah eine Freizügigkeit für Arbeitskräfte in der Montanindustrie vor. Sie blieb allerdings dadurch beschränkt, dass die Nationalstaaten deren Bewegung nach ihren jeweiligen Arbeitsmarktinteressen steuern konnten. Eine weitergehende Freizügigkeitsregelung beschlossen die sechs Vertragsstaaten der EGKS erst im Rahmen der „Römischen Verträge“ von 1957, mit denen die EWG gegründet wurde.

Die EWG-Vertragspartner begründeten die Gewährung der Freizügigkeit, das Argument des klassischen Liberalismus des 19. Jahrhunderts aufgreifend, mit ihrer Bedeutung für das Wirtschaftswachstum und die Angleichung des Wohlstandsniveaus der Mitgliedstaaten. Ziel der europäischen Integration sei die möglichst ungehinderte Zirkulation von Waren, Dienstleistungen, Kapital und Arbeitskräften. Die Initiative für die Freizügigkeitsregelung ging erneut, wie schon im Falle der EGKS, von Italien aus. Für das südeuropäische Abwanderungsland bildete die Freizügigkeit eines der Kernelemente seiner Europapolitik.

Während bei den EGKS-Verhandlungen die italienische Auffassung noch auf erheblichen Widerstand der Vertragspartner gestoßen war, vermochte Italien 1957 seine Position weitgehend durchzusetzen. Im Hintergrund stand der stark gestiegene Arbeitskräftebedarf in den fünf Partnerländern seit Anfang der 1950er Jahre. Selbst in der Bundesrepublik Deutschland, die 1951 noch eine hohe Erwerbslosigkeit registrierte, herrschte seit 1956 Vollbeschäftigung, weshalb auf den ersten bundesdeutschen Anwerbevertrag mit Italien 1955 seit 1960 weitere mit Spanien, Griechenland, der Türkei, Marokko, Portugal, Tunesien und schließlich mit Jugoslawien folgten. Dennoch übernahmen die anderen EWGMitgliedstaaten die italienische Position nicht in vollem Umfang: Um in der Lage zu sein, jederzeit die eigenen Arbeitsmärkte zu schützen, wurde den EWG-Staaten 
die Möglichkeit eingeräumt, ,aus Gründen der öffentlichen Ordnung, Sicherheit und Gesundheit" die Freizügigkeit zu begrenzen. ${ }^{1}$ Unterschiedliche nationale Interessen in der Freizügigkeitsfrage verschränkten sich folglich in den Römischen Verträgen. Italien blieb in den Folgejahren ein wesentlicher Antreiber für die Implementierung der Freizügigkeitsregelung, die zunächst auch weiterhin vor allem von italienischen Migrantinnen und Migranten genutzt wurde (Romero 2015, S. 33).

Artikel 48 des EWG-Vertrags von 1957 legte fest, dass die Freizügigkeit für Arbeitskräfte innerhalb der Gemeinschaft bis 1969 durchzusetzen sei. Danach habe jede ,unterschiedliche Behandlung der Arbeitnehmer der Mitgliedstaaten in Bezug auf Beschäftigung, Entlohnung und sonstige Arbeitsbedingungen“ zu unterbleiben. ${ }^{2}$ Auch durch die Zusammenarbeit der nationalen Arbeitsverwaltungen sollten Hemmnisse der Bewegung zwischen den Mitgliedstaaten abgebaut und ein europäischer Arbeitsmarkt durch den Ausgleich von Angebot und Nachfrage nach Arbeitskräften bzw. nach Arbeit hergestellt werden. Die drei Freizügigkeitsverordnungen von 1961, 1964 und 1968 setzten dieses Vorhaben der Römischen Verträge um, erleichterten eine „europäische Binnenwanderung“ von Arbeitskräften (und von ihren Familienangehörigen) und erschwerten eine nationale Kontrolle der jeweiligen Arbeitsmärkte. Die Verordnung von 1961 gab die Arbeitsaufnahme in einem anderen Mitgliedsstaat grundsätzlich frei und hob die Visumpflicht auf, ein Personalausweis oder ein Reisepass legitimierten das Überschreiten der Grenze. 1964 folgte die Aufhebung des Vorrechts für einheimische Arbeitskräfte bei der Vermittlung von Arbeitsplätzen. Seit 1968 schließlich benötigten Arbeitsmigrantinnen und Arbeitsmigranten innerhalb der EWG keine nationale Arbeitserlaubnis mehr (Goedings 2005).

Die Regelungen von 1961 und 1964 sahen zwar noch zahlreiche Beschränkungen der Freizügigkeit durch nationale Eingriffsmöglichkeiten vor. Sie wurden aber aufgrund des hohen Arbeitskräftebedarfs der Ökonomien in Belgien, der Bundesrepublik Deutschland, Frankreich, Luxemburg und den Niederlanden in der Regel nicht genutzt. Das heißt: Faktisch herrschte für Arbeitskräfte aus Staaten der EWG bereits seit 1961 eine weitgehend uneingeschränkte Möglichkeit, Arbeit in den anderen Mitgliedstaaten zu suchen und $\mathrm{zu}$ finden, in das Herkunftsland zurückzukehren oder den Arbeitsplatz innerhalb des EWG-Raums zu wechseln, um möglichst gute Arbeits- und Lohnbedingungen zu erreichen (Sparschuh 2019, S. 97-106).

\footnotetext{
${ }^{1}$ Artikel 56 des EWG-Vertrags, https://ec.europa.eu/romania/sites/romania/files/tratatul_de_ la_roma.pdf (04.09.2020).

${ }^{2}$ https://ec.europa.eu/romania/sites/romania/files/tratatul_de_la_roma.pdf (04.09.2020).
} 
In den Gründungsdokumenten der EWG bezogen sich die Freizügigkeitsregelungen ausschließlich auf ökonomisch aktive Frauen und Männer und ihre Familienangehörigen. In den 1970er Jahren sorgte vor allem die Rechtsprechung des 1952 gegründeten Europäischen Gerichtshofs dafür, dass sie auch auf andere Menschen in Bewegung, darunter Studierende, Nicht-erwerbstätige oder Personen im Rentenalter ausgeweitet wurden (Rogers et al. 2012, S. 30-46). Die Urteile sind später in Verordnungen der EWG/EG überführt worden. Davon ausgehend etablierte sich die Freizügigkeit zu einem Kernziel der EU, das schließlich 1992 bei der Gründung der EU im ,Vertrag über die Arbeitsweise der Europäischen Union“" auch in den Rechtekanon der „Unionsbürger“ eingeführt wurde. Alle Unionsbürgerinnen und Unionsbürger verfügen laut Artikel 20 über ,,das Recht, sich im Hoheitsgebiet der Mitgliedstaaten frei zu bewegen und aufzuhalten“. 3

\footnotetext{
${ }^{3}$ https://eur-lex.europa.eu/legal-content/EN/TXT/PDF/?uri=CELEX:12012E/TXT\&fro $\mathrm{m}=\mathrm{DE}(04.09 .2020)$.
} 
Open Access Dieses Kapitel wird unter der Creative Commons Namensnennung 4.0 International Lizenz (http://creativecommons.org/licenses/by/4.0/deed.de) veröffentlicht, welche die Nutzung, Vervielfältigung, Bearbeitung, Verbreitung und Wiedergabe in jeglichem Medium und Format erlaubt, sofern Sie den/die ursprünglichen Autor(en) und die Quelle ordnungsgemäß nennen, einen Link zur Creative Commons Lizenz beifügen und angeben, ob Änderungen vorgenommen wurden.

Die in diesem Kapitel enthaltenen Bilder und sonstiges Drittmaterial unterliegen ebenfalls der genannten Creative Commons Lizenz, sofern sich aus der Abbildungslegende nichts anderes ergibt. Sofern das betreffende Material nicht unter der genannten Creative Commons Lizenz steht und die betreffende Handlung nicht nach gesetzlichen Vorschriften erlaubt ist, ist für die oben aufgeführten Weiterverwendungen des Materials die Einwilligung des jeweiligen Rechteinhabers einzuholen. 